\title{
Cultura investigativa científica: una propuesta para el desarrollo del estado del arte en estudiantes de educación media general
}

\author{
Scientific research culture: a proposal for the development of the \\ state of the art in general students of high school
}

Lipselotte de Jesús Infante Rivera ${ }^{1}$

1 Universidad Rómulo Gallegos, Venezuela

\section{RESUMEN}

El presente estudio tuvo como objetivo proponer acciones para el desarrollo del estado del arte en los estudiantes de Educación Media General. Se utilizó una investigación de tipo descriptiva, bajo la modalidad de proyecto factible. La muestra del estudio fue probabilística que incluyó a 84 estudiantes de 5to año de bachillerato a los cuales se les aplicó un instrumento de 25 ítems, diseñado a través de las variables en estudio, el cual tuvo una consistencia de 0,93\% en la correlación de los ítems, el cual se realizó mediante el coeficiente Alfa de Cronbach. La discusión de los resultados contribuyó a determinar que los estudiantes de bachillerato, aun no tienen la noción de cómo y dónde investigar, lo que indica falta de orientación por parte de los docentes que imparten las asignaturas relacionadas con metodología. Entre las conclusiones más destacadas se obtuvo que los estudiantes en su gran mayoría no conocen el método científico, ni la forma adecuada de redactar informes o simples trabajos de sus asignaturas, y los docentes no se encuentran preparados para contribuir al desarrollo de una cultura investigativa. Por tanto, la propuesta para realizar actividades de actualización docente puede contribuir en función de que obtengan un conocimiento y competencias necesarias para impartir el aprendizaje que se espera en cuanto a la realización de proyectos y formación de cultura investigativa en los estudiantes.

Palabras clave: Cultura investigativa, evaluación, estado del arte, educación.

\begin{abstract}
The objective of this study was to propose actions for the development of the state of the art in students of General Media Education. A descriptive research was used, under the feasible project modality. The sample of the study was probabilistic that included 84 students of 5 th year of high school, to which a 25 item instrument was applied, designed through the variables under study, which had a consistency of $0,93 \%$ in the correlation of the items, which was done by the Cronbach's Alpha coefficient. The discussion of the results helped to determine that the high school students, still do not have the notion of how and where to investigate, which indicates lack of guidance on the part of the teachers who teach the subjects related to methodology. Among the most important conclusions was that the vast majority of students do not know the scientific method, nor the proper way to write reports or simple works of their subjects, and teachers are not prepared to contribute to the development of a research culture. Therefore, the proposal to carry out teacher refresher activities can contribute in order to obtain knowledge and skills necessary to impart the learning expected in terms of conducting projects and training in research culture in students.
\end{abstract}

Keywords: Investigative culture, evaluation, state of the art, education.

Historial del artículo:

Recibido, 05 de junio de 2017; aceptado, 18 de junio de 2017; disponible en línea, 30 de junio de 2017

* Profesora en Educación Mención Integral de la Universidad Pedagógica Experimental Libertador; Magíster Scientiarum en Educación Mención Desarrollo Comunitario. y Magíster Scientiarum en Educación Mención Investigación Educativa.

Correo: infantelipselotte038@gmail.com 


\section{INTRODUCCIÓN}

Hoy en día, los estudiantes de Educación Media General, sobre todo los que cursan el 5to año, se encuentran en una disyuntiva para la elaboración del proyecto científico que han de realizar para egresar ese nivel; ya que es un requisito emanado por entes superiores del Ministerio del Poder Popular para la Educación con lineamientos que en muchas ocasiones ni siquiera los mismos docentes se encuentran preparados para impartir, a sabiendas de que le corresponde a la educación desde niveles anteriores al bachillerato, formar a los estudiantes para que puedan tener un poco de conocimiento en cuanto a la realización de un trabajo de investigación. Esa discusión sobre el docente no es más que una parte de la problemática, puesto que son un conjunto de elementos los que en realidad ejercen influencia en este aspecto.

Se observa que la tecnología se ha interpuesto por encima de los valores investigativos del individuo, comenzando por la forma tan evidente que tienen los estudiantes de entregar en sus trabajos una copia de otros que están colgados en la internet, trayendo como consecuencia que estos no desarrollen su capacidad creativa ni el pensamiento lógico, coherente y funcional que apuesta por respuestas de envergadura a necesidades del entorno.

Resulta oportuno hablar sobre la cultura investigativa, de la cual carecen la mayoría de los estudiantes en la actualidad, por esto mismo de la falta de formación que se evidencia al no contar con hallazgos investigativos de relevancia en los trabajos de dichos estudiantes. Y, es que, no hay perfil en los docentes para tales fines, es decir, no se abordan temas de relevancia que motiven a que el joven investigue y adquiera las competencias necesarias para llevar a cabo estudios con aportes de relevancia.

El presente estudio argumenta, la importancia de influenciar en la cultura investigativa de los jóvenes estudiantes, ya que esto les va a permitir una transición hacia la educación universitaria más agradable, en donde el estudiante pueda con mayor facilidad aportar ideas en sus investigaciones, que no sean una copia de otros trabajos, o haga los suyos solo como un requisito para aprobar; en este aspecto, el sentido de pertenencia juega un papel fundamental, ya que por su interés en los actos educativos que ejecute, muchos le van a salir tal y como lo espera. Esto es realmente lo que se requiere para el estudiante actual, los elementos que en realidad le van a ser útil para su futuro y el de los demás.

Es importante destacar, que fue a través de la investigación que los avances de la ciencia han sido posibles, se habla de lo tangible, que se puede comprobar; se habla pues del positivismo que fue una corriente de pensamiento que marcó la primacía del método científico como el único válido para adquirir conocimientos.

Venezuela, a través de su historia demuestra que la investigación científica no ha sido una cultura que genera los mayores productos en su haber, ya que las cifras han fijado al país en uno de los lugares más bajos en investigadores en relación a la población existente. En este sentido, Martínez (2012) refiere que el estimado de investigadores por millón de habitantes para el año 2010 era de 310. Lo que quiere decir, que otros países de Latinoamérica están por encima de lo que Venezuela demuestra en las bases de sus investigadores, y se necesitan muchos más por millón de habitantes para ir a la par de países en vías de desarrollo o ya desarrollados e ir a la vanguardia de las inquietudes investigativas que contribuyan al desarrollo pleno de la sociedad.

De acuerdo a la Comisión Económica para América Latina y el Caribe CEPAL (2006) la inversión y presupuesto del Estado en los Programas de Investigación juega un papel relevante, el gasto en Investigación y Desarrollo en Venezuela era del 0,2 \% al 0,5\% del PIB. Se observa la decadencia en relación a la investigación y el gasto que se presupuesta para tal fin, que no es ni siquiera la primera parte de lo que corresponde para proyectarse como un país de cultura investigativa valiosa.

Esta situación se plantea desde una parte muy puntual como es el caso de los estudiantes de 5to año de media general de los liceos públicos y privados de la ciudad de Upata Municipio Piar, los cuales se encuentran realizando el proyecto de investigación científica a fin de resolver problemáticas a nivel socioeducativo, en fin, se trata de un proyecto que contribuya a mejorar la calidad de vida del individuo y a su vez refuerce los vínculos entre el estudiante y la comunidad.

Aunque el problema radica desde un nivel de aprendiz, es decir, desde el ler año de educación media o cuarto año de bachillerato como es mejor conocido, puesto que a esta edad ya deberían estar impartiéndoseles a los estudiantes las técnicas de investigación acordes con el curso y edad de dichos estudiantes, con el propósito de ir creando la cultura que tan necesaria es para los siguientes grados. En tal sentido, el objetivo general de la presente investigación fue proponer una cultura investigativa científica para el desarrollo del estado del arte en los estudiantes de educación Media General del L.N "Tavera Acosta" Municipio Piar del Estado Bolívar.

De la misma forma, se trazaron los siguientes objetivos específicos, diagnosticar el estado del arte de los estudiantes de educación media general del 
Liceo Nacional "Tavera Acosta" Municipio Piar del Estado Bolívar, determinar la cultura investigativa de los docentes en su desempeño como facilitadores de aprendizaje de los diferentes métodos investigativos para los proyectos científicos, establecer estrategias para una mejor disposición por la cultura investigativa en los estudiantes de 5to año y finalmente, diseñar acciones que le permita a los estudiantes y docentes la adquisición de nuevos conocimientos y formación de una cultura investigativa a través de los diferentes métodos de investigación.

La importancia de la investigación viene dada por la falta de cultura en investigación científica de los estudiantes, en función de la construcción de conocimientos que le permitan tener una cosmovisión del mundo sin limitantes y sin que esto represente un problema acerca de lo que es verdadero, ya que en este proceso de globalización, es necesario poseer una cultura de investigación en donde se vean reflejados los éxitos del desempeño en el campo social y además de la formación de ellos mismos como estudiantes que respondan verdaderamente a las necesidades sociales, políticas, educativas y culturas del país, enmarcadas dentro de una realidad global e integrada a través de un componente investigativo en donde se haga un análisis de los desafíos que poseen los estudiantes en este aspecto para que integren el conocimiento de forma sistémica y redunden en la calidad educativa, además de participar en eventos científicos.

Esta investigación coadyuva en el análisis del estado del arte con respecto a la cultura de investigación y además proponer estrategias que le permitan al estudiante de Educación Media General, aprehender esos conocimientos que acompañan una apropiación crítica y contextualizada a través de la implementación de las habilidades, aprovechando las potencialidades de cada uno para tener los argumentos propios que precisen las respuestas en virtud de lo aprehendido en ese camino y acceso a la investigación como tal. Por otro lado, existe en torno a este estudio la parte ética tanto del docente como del estudiante, ya que el primero tiene en su perfil un arbóreo concentrado axiológico que le permite encaminarse con el mejor comportamiento posible.

Es evidente que el fenómeno abordado ha de constituirse con mayor ahínco en función de los hechos y el producto del mismo. En ese sentido, la importancia de diseñar una propuesta a través de acciones planificadas, es sin dudas una remuneración al trabajo de los docentes para una mayor internalización de los procesos investigativos. Asimismo, bajo la premisa de que la generación actual tiene más posibilidades de hacer investigación que otros de épocas anteriores, se establecen aquí la promoción de actividades en donde se exponen las etapas de: motivación, desarrollo, ejecución y evaluación.
En relación a esto, Pérez (2014) presentó un estudio titulado: Los alumnos como investigadores en el desarrollo de la química de 2 do año de bachillerato. El objetivo principal del estudio fue familiarizar a los estudiantes de bachillerato con el método científico e incrementar la experimentación, investigación y expectativas del alumnado en el campo de la química por medio de experimentos y actividades vinculadas a experiencias cotidianas y que refuerzan el curriculum del curso.

Roso (2012) presentó una investigación la cual llevó por título: Investigación en Enseñanza Secundaria: los "jóvenes investigadores", El objetivo de esta investigación fue colaborar en el proceso de enseñanza/aprendizaje desde una óptica distinta y complementaria a la establecida por el propio sistema y currículo académico en el I.E.S. Mario Roso de Luna de Logrosán (Cáceres). Este proyecto se inició para los "Jóvenes investigadores" con enfoque didáctico adecuado y adaptado a los alumnos/as existentes en la Comarca de las Villuercas (Cáceres).

En el mismo orden de ideas, Charro, Gómez, Plaza y Padilla (2013) presentaron una investigación titulada: Un estudio Delphi para descubrir cómo Intensificar, Mejorar y popularizar la cultura científica en los alumnos de secundaria y Bachillerato, en la Universidad de Valladolid cuyo objetivo principal fue identificar los puntos clave en los distintos campos de las ciencias que pueden mejorar la cultura científica en la sociedad actual.

También Álvarez, Figuera y Torrado (2011) presentaron un estudio en la Universidad de Barcelona, el cual titularon: La problemática de la transición bachilleratouniversidad en la Universidad de Barcelona. El propósito de este estudio fue presentar algunos datos sobre el perfil personal y académico del estudiante que abandona o bien continúa sus estudios al término del segundo año y, al mismo tiempo, la valoración que hacen estos estudiantes que continuaban del proceso de la transición.

Zárate (2009) presentó un estudio titulado: Estrategias de enseñanza para desarrollar habilidades del pensamiento en la Escuela Básica Estadal Caura, para la Universidad Nacional Experimental de Guayana. El objetivo de dicha investigación fue proponer estrategias de enseñanza para desarrollar habilidades del pensamiento en los alumnos de la Escuela Básica Estadal Caura en Puerto Ordaz Estado Bolívar.

Atendiendo a la problemática planteada, en éste estudio se proponen acciones que le permiten tanto al docente como al estudiante avanzar en el sentido y dirección de la cultura investigativa, haciendo énfasis en su conocimiento previo y el estado del arte que estos poseen sobre la investigación científica, de 
esta forma se puede contribuir a que se pueda ver el método científico como el principal aporte a la sociedad y los avances que se han tenido en función de dar respuestas a las necesidades de los individuos. Es por esto que se considera como factor elemental en la formación del estudiante desde muy temprana edad, es decir, que se habitúen a hacer investigación y además que tengan las habilidades necesarias para enfrentar los retos de estudios superiores.

En el estudio no se presentaron dificultades o limitaciones mayores que intervinieran en el proceso investigativo como tal, ya que se pudo recolectar la información de manera tal que se obtuvieron datos importantes respecto de la muestra seleccionada.

\section{MATERIAL Y MÉTODOS}

La metodología en la cual se enmarcó el presente trabajo de investigación fue de campo, en función de recoger los datos en el escenario donde se suscitaron los hechos, además, se trabajó mediante un enfoque cuantitativo, para dar cabida al método científico que es el que se corresponde a la evaluación del estado del arte para conocer sobre la cultura investigativa tanto de los jóvenes estudiantes como de los docentes.

Para esto la población estuvo conformada por 107 elementos que fueron los estudiantes de ambos géneros de 3 secciones de 5to año de bachillerato del Liceo Nacional "Tavera Acosta" a partir de allí se seleccionaron 84 elementos a través de una muestra probabilística, a los cuales se les aplicó un instrumento que abarcó las variables establecidas en la operacionalización de variables con una escala de Likert de 25 ítems con una fiabilidad de 0,93\% de acuerdo al modelo de consistencia Alfa de Cronbach, con criterios de selección que se codificaron en el software SPSS 22 como: Muy de acuerdo, de acuerdo, ni de acuerdo, ni en desacuerdo, en desacuerdo y muy en desacuerdo.

Para este procedimiento se aplicaron los ítems correspondientes, en donde se midieron las siguientes variables: conocimiento sobre metodología (teórico - práctico), el desempeño docente (personal y profesional), estrategias (Didácticas y Dinámicas) y habilidades (Cognitivas y Técnicas). Se puede observar a cada variable con las dimensiones correspondientes.

\section{RESULTADOS}

Se presentan los resultados en forma de tablas y gráficos con sus porcentajes, a fin de proporcionar organización y evidenciar los hallazgos encontrados en la investigación, conectándolos con algunos sustentos teóricos cuando así lo requiera la respuesta proporcionada o a consideración del investigador, estableciendo una conexión lógica entre las respuestas, que fueron las que contribuyeron a extraer la opinión de los estudiantes y docentes en el Liceo Nacional "Tavera Acosta" acerca del estado del arte y la cultura investigativa científica de los mismos. A continuación, se detallan los resultados de las variables:

Variable: conocimiento sobre metodología

Se observa en la tabla 1 que el $75 \%$ de los estudiantes

Tabla 1

La investigación científica es importante para dar respuesta a las necesidades del individuo.

\begin{tabular}{lcr}
\hline \multicolumn{1}{c}{$\begin{array}{c}\text { Criterios de } \\
\text { selección }\end{array}$} & Frecuencia & Porcentaje \\
\hline Muy de acuerdo & 11 & $113,1 \%$ \\
De acuerdo & 63 & $75,0 \%$ \\
Ni de acuerdo, ni & 5 & $6,0 \%$ \\
en desacuerdo & & \\
En desacuerdo & 5 & $6,0 \%$ \\
Total & 84 & $100,0 \%$ \\
\hline
\end{tabular}

encuestados se encuentran de acuerdo en que la investigación es importante, al igual que el 13,1 \% los cuales se expresaron en la misma línea al estar muy de acuerdo. Se evidencia que la investigación constituye una herramienta lucrativa a favor de los estudiantes, ya que es la que va a contribuir en su desarrollo y formación académica y profesional a posteriori. Solo un pequeño porcentaje del $6 \%$ se encuentran desinteresados por esto y el resto ni de acuerdo ni en desacuerdo.

En este orden de ideas, Sampieri, Fernández y Baptista (2014) refieren que la investigación es un conjunto de procesos sistemáticos, críticos y empíricos que se aplican al estudio de un problema o fenómeno. En la actualidad es muy importante que el estudiante aprenda a investigar, a fin de garantizar en estos tiempos de

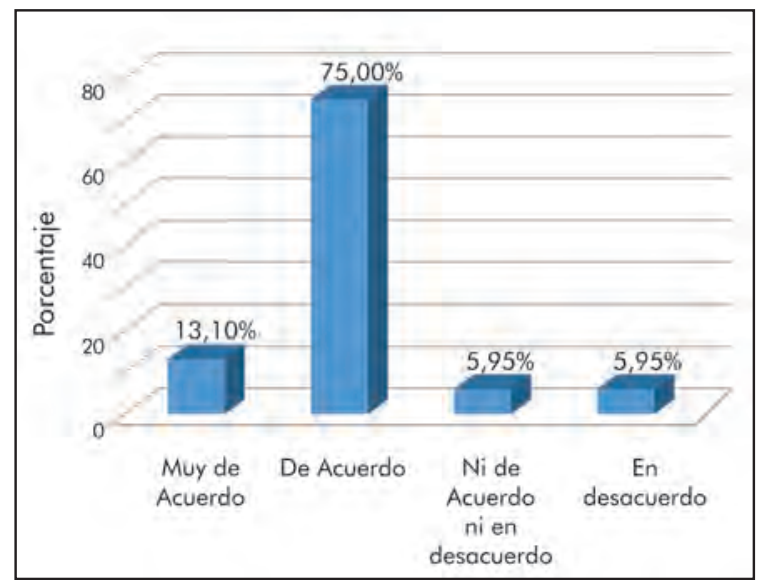

Figura 1. Importancia de la investigación científica. 
globalización, ventajas que lo ayuden a destacarse en cualquier ámbito donde desee desempeñarse.

Tal como se puede evidenciar en la tabla 1, existe una relación importante en cuanto a los que están de acuerdo con que la investigación científica es, de hecho, muy significativa para responder a las necesidades de la vida diaria, y por el contrario un porcentaje del 5,95 $\%$ refirió no estar de acuerdo ni en desacuerdo al igual que el resto. Puede decirse, en torno a los resultados, que existe un auge en ésta sociedad del conocimiento, y en razón de eso, el docente ha de estar preparado para impartir aspectos que fundamenten el desarrollo y cultura por la investigación, para de esta forma alcanzar los avances tecnológicos y científicos los cuales formulan uno de los poderes fundamentales en la actualidad.

\section{Variable: Estrategias}

Se ilustra en la tabla 2 , que la mayoría de los estudiantes representados por el 78,6\% están es desacuerdo en que los docentes aplican las evaluaciones relacionadas al tema de la asignatura que imparte y el 14,3\% estuvieron muy en desacuerdo.

Con respecto a la figura 2, correspondiente a la variable: estrategia, se puede observar en porcentajes elevados de $78,57 \%$ y $14,29 \%$ en los criterios de:

Tabla 2

Se aplican las evaluaciones de acuerdo a las actividades y temas de cada asignatura.

\begin{tabular}{lcc}
\hline \multicolumn{1}{c}{$\begin{array}{c}\text { Criterios de } \\
\text { selección }\end{array}$} & Frecuencia & Porcentaje \\
\hline Ni de acuerdo, ni & 6 & $7,1 \%$ \\
en desacuerdo & & \\
En desacuerdo & 66 & $78,6 \%$ \\
Muy en desacuerdo & 12 & $14,3 \%$ \\
Total & 84 & $100,0 \%$ \\
\hline
\end{tabular}

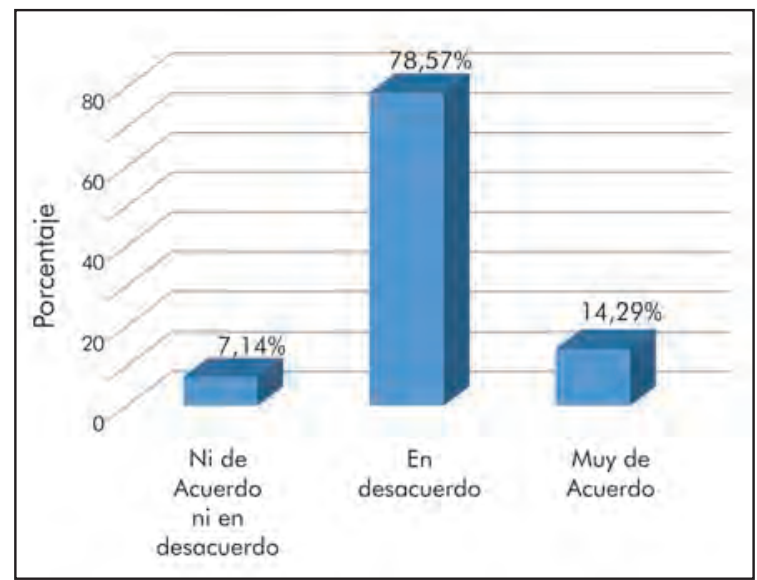

Figura 2. Evaluaciones y actividades sobre los temas de cada asignatura. en desacuerdo y muy en desacuerdo, respectivamente, la desaprobación de los estudiantes en cuanto a las evaluaciones que se realizan, las cuales no se corresponden con las actividades o temas de la asignatura. En ese sentido se puede decir, que la evaluación es un elemento clave en la educación, por tanto, el docente ha de tener una planificación de cada estrategia evaluativa para los diferentes temas y contenidos de las asignaturas, esto demuestra eficiencia y eficacia por parte del docente, en caso contrario, demuestra que el docente no posee las competencias para diseñar instrumentos de evaluación acorde a su planificación.

\section{Variable: Habilidades}

Como puede apreciarse en la tabla 3 , un porcentaje representado por 40,5\% en los criterios: en desacuerdo y muy en desacuerdo refieren que los docentes no poseen las competencias para poner en práctica proyectos de investigación científica. Esto genera una problemática a la hora de conformar el proyecto, ya que los estudiantes no van a tener los conocimientos que requieren para realizar el trabajo.

La figura 3 expresa el desacuerdo de los estudiantes encuestados, los cuales son reflejados por porcentajes

Tabla 3

Los docentes poseen competencias para la conformación y puesta en práctica de los proyectos científicos.

\begin{tabular}{lcr}
\hline \multicolumn{1}{c}{$\begin{array}{c}\text { Criterios de } \\
\text { selección }\end{array}$} & Frecuencia & Porcentaje \\
\hline De acuerdo & 6 & $7,1 \%$ \\
Ni de acuerdo, ni & 10 & $11,9 \%$ \\
en desacuerdo & & \\
En desacuerdo & 34 & $40,5 \%$ \\
Muy en desacuerdo & 34 & $40,5 \%$ \\
Total & 84 & $100,0 \%$ \\
\hline
\end{tabular}

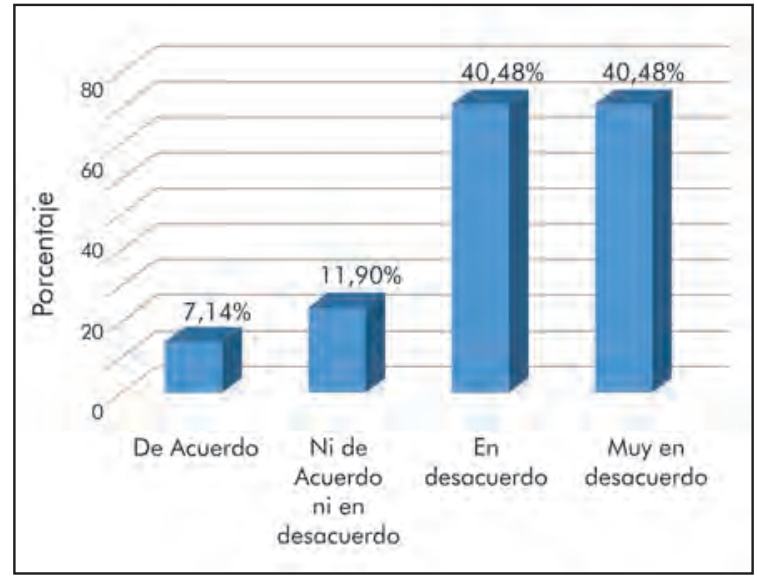

Figura 3. Competencias docentes. 
iguales del 40,48\% en los criterios correspondientes. El $11,90 \%$ no estuvo de acuerdo ni en desacuerdo y solo un mínimo porcentaje del 7,14 \% estuvo de acuerdo con que los docentes poseen las competencias para la puesta en práctica de los proyectos científicos, lo que dista de la realidad reflejada por el porcentaje mayor. A raíz de estos resultados, se hace necesario abordar la formación docente en este aspecto, ya que existe la necesidad de que los estudiantes egresen con una excelente formación y cultura investigativa.

\section{DISCUSIÓN}

La investigación siempre ha sido el horizonte del ser humano, éste ha investigado desde sus inicios, logrando situaciones que lo han catapultado hasta esta sociedad del conocimiento en la cual se vive en la actualidad. Siempre busca un motivo, una razón y una verdad; en suma, siempre está investigando. Hay que recordar también, que la mayor parte de los inventos y tecnologías en el mundo, de una u otra forma han sido producto de la investigación, ya sea por estar más cómodos o por dar respuestas a las necesidades y carencias del ser humano, ejemplo de ello pueden ser las viviendas, los automóviles, teléfonos móviles y muchas otras invenciones que han traído consigo la solución a problemáticas existentes.

La investigación del estudiante como un proceso de aprendizaje está fundamentada en la exploración y en la capacidad para el pensamiento racional, así como en los rasgos fundamentales del quehacer científico, por lo que es relevante que se haga el esfuerzo para que estos adquieran las herramientas que le permitan avanzar adecuadamente hacia otros estudios, pudiendo de esta forma dar respuestas a problemáticas de su entorno. Se desea que sea ese estudiante quien tenga la creatividad de diseñar proyectos científicos en beneficio de la colectividad; pero para ello, debe tener el conocimiento en cuanto a métodos, enfoques y otras orientaciones que se requieren para entrar al campo de estudio y abordar un fenómeno.

En relación a la discusión de los resultados sobre el conocimiento de los estudiantes de 5to año, se pudo evidenciar que su estado del arte es poco o nulo con respecto a la investigación científica, y no poseen una cultura investigativa que los proyecte como baluartes de su entorno. A estos individuos se les aplicó un instrumento con varios criterios de forma escala de Likert, en donde quedó claro que muchos de ellos están de acuerdo con los planteamientos que se les hizo. Para ellos las páginas de internet son una fuente confiable para investigar y sustentar teóricamente los proyectos y trabajos que deben entregar, además de adquirir nuevos conocimientos.

En función de esto, es preciso acotar, que los estudiantes a esta edad en la cual aún transitan por el bachillerato, carecen de la pericia necesaria de cómo y dónde investigar, por lo que se hace necesario que sean los docentes quienes conduzcan ese aprendizaje por medio de tips que les permitan avanzar tanto en conocimiento como en habilidades.

Los estudiantes que asumieron una postura en la cual estaban en desacuerdo o muy en desacuerdo con los planteamientos, y se representan con un alto porcentaje de estos, todo lo cual conlleva a interpretar que los docentes carecen de competencias para impartir áreas de conocimiento relacionadas a la ciencia, por lo que se debe tomar en consideración los diferentes programas de formación para poder multiplicar la información sobre los proyectos científicos de manera ecuánime y veraz.

La cultura investigativa aporta al ser humano herramientas que conllevan a dar respuestas a fin de lograr objetivos de necesidades de su entorno. Esto, por cuanto se puede contar con un individuo que sienta motivación por acercarse al mundo de lo desconocido para buscar la razón de ser de estas.

Se observó que, en algunos estudiantes no existe motivación para investigar y que las evaluaciones no se corresponden a los temas y contenidos de la planificación del docente. Asimismo, se pudo conocer que los estudiantes en su gran mayoría no conocen el método científico, la forma adecuada de redactar informes, ni simples trabajos de sus asignaturas.

Utilizan páginas de internet para hacer investigaciones, las cuales no poseen ninguna credibilidad y casi todos coinciden en las páginas de búsquedas, trayendo como consecuencia que lleven las mismas informaciones. se pudo conocer, además que los docentes no se encuentran preparados para contribuir al desarrollo de una cultura investigativa.

Todos estos hallazgos permitieron llegar a las conclusiones de que:

1 El estado del arte de los estudiantes es poco o nada en relación a los métodos y formas de investigación, lo que incide en la participación de los mismos en los proyectos de investigación y, además, en el rendimiento académico de áreas del conocimiento.

2 Se deben desarrollar e implementar estrategias para que los estudiantes puedan construir esquemas de conocimiento, que les permitan adquirir una visión de la realidad superior a un "saber cotidiano" y los acerquen al "conocimiento elaborado en la comunidad científica".

De acuerdo a cada objetivo se pudo evidenciar, en el primero para diagnosticar el estado del arte de los estudiantes de educación media general del Liceo 
Nacional "Tavera Acosta" Municipio Piar del Estado Bolívar, se realizaron visitas de observaciones de acuerdo al tiempo de docentes y estudiantes, a fin de garantizar encuentros de calidad. Asimismo, se establecieron etapas en el plan de acción para orientar de mejor forma cada uno de los pasos a seguir.

Para el segundo objetivo, que fue determinar la cultura investigativa de los docentes en su desempeño como facilitadores de aprendizaje de los diferentes métodos investigativos para los proyectos científicos, se aplicó el instrumento de recolección de información, desde donde se obtuvieron los hallazgos de la investigación y las posteriores acciones de acuerdo a cada etapa cumplida del plan de acción.

En el tercer objetivo, establecer estrategias para una mejor disposición por la cultura investigativa en los estudiantes de 5to año, se dispuso el diseño de un plan de acción que recogiera actividades inherentes al proceso de formación de los docentes a través de talleres, charlas y diálogos. De la misma forma se tomó en cuenta a los estudiantes para dirigir talleres que contribuyeran a establecer una cultura investigativa a través del desarrollo del estado del arte.

El cuarto y último objetivo, que fue diseñar acciones que le permita a los estudiantes y docentes la adquisición de nuevos conocimientos y formación de una cultura investigativa a través de los diferentes métodos de investigación. Esto permitió contar con estrategias que permitieran la adquisición de competencias tanto a docentes como a estudiantes, y es en suma un alcance de todos y cada uno de los objetivos, por cuanto en la etapa de evaluación del plan se pueden observar grandes logros.

Los resultados de los autores consultados coinciden con los hallazgos de esta investigación, ya que, se observa en Roso que los alumnos/as se convierten en los sujetos activos de una "investigación", cuya temática e interés no viene de otros ámbitos educativos sino de sus propias inquietudes.

Pérez, por su parte expresa que la educación es un valor que debe promoverse dentro y desde fuera de las aulas, y que, a su vez, mejora la inclusión social. Es necesaria la participación y colaboración de todos, ya que ha cambiado la figura del profesor que necesita trabajar en equipo, contando con el entorno familiar, comunitario, cultural y socio laboral, el asociacionismo y los medios de comunicación.

En ambos casos de las conclusiones se derivan los aspectos positivos que tiene: la importancia de que sea el estudiante el promotor de su propia investigación, y el docente su guía en la consecución de los objetivos planteados y además investigar fuera de las aulas en un ambiente comunitario, reforzando de esta forma los lazos con la comunidad y el sentido de pertenencia que trae consigo la cercanía con las necesidades y la motivación o interés de dar respuestas a las mismas.

De acuerdo con Charro y otros, la cultura científica se puede lograr $\circ$ mejorar de acuerdo a los intereses particulares y hábitos de cada persona. En ambos estudios concuerdan en que por estudiar la cultura investigativa científica se pretende lograr un fundamento o más bien un hábito que contribuya en la formación del verdadero estudiante, ese que requiere la sociedad actual para dar respuestas a las necesidades del entorno.

En lo que respecta al estudio de Álvarez, Figuera y Torrado hay una correspondencia entre la presente investigación y la de los autores por cuanto se estudia la transición y el cambio que muchas veces se torna en algo difícil de sostener. Es por ello, que la preparación - formación que traigan como base el estudiante desde el bachillerato a la universidad es primordial, y debe estar enmarcada dentro de una serie de elementos básicos como el interés por formarse con vocación y no por opción y la motivación por ser cada día mejor en lo que quiera o pretenda desempeñarse. Estos dos elementos son indispensables, y es a lo que se pretende llegar con el presente estudio, haciendo énfasis en el interés que por orientación vocacional ha de estar adherido en la concepción del nuevo bachiller. Lo anterior también se relaciona ésta investigación con el estudio de Álvarez, Figuera y Torrado.

La investigación que presentó Zarate puede dar cuenta de las competencias que necesitan los estudiantes para desarrollar sus habilidades de pensamiento, todo lo cual fue encausado por la investigadora para proponer estrategias que contribuyeran para tal fin. La teoría de donde se sustento fue la de Beyer (1998), el cual refiere sobre el desarrollo de habilidades del pensamiento, así como los aportes de Vygotsky sobre el aprendizaje cooperativo. En ese sentido, se relaciona con la presente investigación ya que se trata de desarrollar habilidades, lo que se corresponde a la propuesta para evaluar el estado del arte, que es el conocimiento que tienen los estudiantes de 5to año, en relación a la cultura investigativa.

\section{Agradecimientos}

En especial a los estudiantes del Liceo Nacional "Tavera Acosta", por tan valiosa contribución en la realización de este trabajo de investigación. 


\section{REFERENCIAS BIBLIOGRÁFICAS}

Charro, E; Gómez, Á; Plaza, S y Padilla, Y (2013). Un estudio Delphi para descubrir cómo Intensificar, Mejorar y popularizar la cultura científica en los alumnos de secundaria y Bachillerato. Recuperado de http://cerro.cpd.uva.es/handle/10324/3768.

Comisión Económica para América Latina y el Caribe CEPAL (2006). La heterogeneidad en el esfuerzo innovador a nivel mundial. Recuperado de https:// www.cepal.org/noticias/paginas/8/33638/ Innovacionparaeldesarrollo.pdf.

Martínez, M (2012). Epistemología. Postgrados Ciudad Bolívar. Presentación del Taller. [Serie de 122 Diapositivas]. Upata-Estado Bolívar. Venezuela.

Pérez, J (2014). Los alumnos como investigadores en el desarrollo de la química de 2 do año de bachillerato. (Tesis inédita de maestría) Universidad de Oviedo. Recuperado de http://dspace.sheol. uniovi.es/dspace/handle/10651/27525.
Roso, M (2010). Investigación en Enseñanza Secundaria: los "jóvenes investigadores". (Tesis inédita de maestría) I.E.S. Mario Roso de Luna (Logrosán), Cáceres. Recuperado de dehesa.unex. es/handle/10662/4628.

Sampieri, Fernández y Baptista, (2014). Metodología de la Investigación. Ciudad de México. Editores McGraw Hill Education.

Zárate, S (2012). Estrategias de Enseñanza para desarrollar habilidades del pensamiento en la Escuela Básica Estadal Caura. Recuperado de www.cidar.uneg.edu.ve/DB/bcuneg/EDOCS/.../ TGMLZ37S882009ZarateSusana.pdf. 